\title{
Diacronie
}

Studi di Storia Contemporanea

$N^{\circ} 28,4 \mid 2016$

La voce del silenzio

\section{Miloš Ković (a cura di), Srbi 1903-1914. Istorija ideja}

I serbi 1903-1914. Storia delle idee

\section{Fabio Giomi}

\section{(2) OpenEdition}

\section{Journals}

\section{Edizione digitale}

URL: http://journals.openedition.org/diacronie/4405

DOI: 10.4000/diacronie.4405

ISSN: 2038-0925

\section{Editore}

Association culturelle Diacronie

\section{Notizia bibliografica digitale}

Fabio Giomi, « Miloš Ković (a cura di), Srbi 1903-1914. Istorija ideja », Diacronie [Online], Nº 28, 4| 2016, Messo online il 29 décembre 2016, consultato il 24 septembre 2020. URL : http://

journals.openedition.org/diacronie/4405; DOI : https://doi.org/10.4000/diacronie.4405 


\title{
Diacronie
}

N. 28 | 4|2016 La voce del silenzio: intelligence, spionaggio e conflitto nel XX secolo

\section{5/}

\section{PANORAMICA: Area post jugoslava 2015}

\author{
Marco ABRAM, Sara BERNARD, Štefan ČOK, Fabio GIOMI, Monica PRIANTE, \\ Francesca ROLANDI, Alfredo SASSO *
}

\author{
TROCH, Pieter, Nationalism and Yugoslavia: Education, Yugoslavism \\ and the Balkans Before World War II, London-New York, I. B. Tauris \& \\ Co., 2015, 320 pp. \\ a cura di Marco ABRAM
}

Un titolo apparentemente generico introduce una monografia destinata a incidere in termini significativi sulla comprensione storiografica dell'esperienza della Jugoslavia monarchica nel periodo tra le due guerre mondiali. Il lavoro raccoglie e sistematizza i risultati delle estese ricerche dedicate da Pieter Troch al controverso processo di nation-building jugoslavo e alla definizione di una comune identità nazionale per i popoli slavo-meridionali. Le politiche culturali ed educative messe in atto nella Jugoslavia monarchica sono state oggetto di alcuni autorevoli studi, tuttavia Troch offre un approccio innovativo che dimostra l'assimilazione delle più recenti tendenze della storiografia culturalista. La monografia prende in considerazione le diverse fasi di vita del Regno - attraverso l'esperienza della dittatura di Re Aleksandar, fino agli anni che precedettero la seconda guerra mondiale - analizzando l'articolazione della dimensione identitaria nazionale jugoslava nei programmi scolastici, in relazione alle forme di appartenenza "tribale" serba, croata e slovena. Vengono quindi indagati i limiti della politica integrativa jugoslava: dalle contraddizioni emerse nell'organizzazione delle celebrazioni scolastiche al ruolo degli insegnanti come principali attori nella dimensione pratica quotidiana. La ricerca si contraddistingue soprattutto per l'accento sulla fluidità e sulla molteplicità delle opzioni identitarie, approfondendo la relazione tra particolarismi e integrazione nazionale e presentandola in comparazione con una più ampia casistica europea. I risultati delle ricerche di Troch incoraggiano la revisione di letture eccessivamente rigide e deterministiche dell'esperienza della Prima 
Jugoslavia, favorendo una più articolata comprensione dei pluriennali tentativi di unificazione slavo-meridionale.

\section{BAKER, Catherine, The Yugoslav Wars of the 199os, London, Palgrave, 2015, 181 pp.}

a cura di Sara BERNARD

I volumi pubblicati da Palgrave nella collana "Studies in European History", di cui il presente studio è il più recente contributo, si pongono tre principali obiettivi: fornire un'accurata e documentata analisi su questioni chiave della storia europea; presentare il punto di vista di un(a) studioso/a coinvolto/a nel dibattito sul tema analizzato; offrire a studenti e docenti un testo sintetico per introdurli al tema e alla rispettiva letteratura. Questo approccio analitico e narrativo emerge chiaramente nel volume dedicato da Catherine Baker alle guerre jugoslave degli anni Novanta.

Nel volume, Baker, specialista di questioni culturali e relazioni internazionali nel post-guerra fredda e autrice di numerosi studi sulla (ri)costruzione di identità culturali e politiche nelle ex repubbliche jugoslave, offre una lucida analisi dello stato dell'arte sulle guerre jugoslave in lingua inglese. In particolare, l'autrice espone con sguardo critico le principali controversie, come quelle relative alle colpe e alle responsabilità per lo scoppio della guerra e alla spinosa questione dell'utilizzo della categoria di genocidio per il massacro di Srebrenica. Inoltre, Baker mostra come negli anni il dibattito sia cambiato grazie all'utilizzo e alla disponibilità di nuove fonti - come la documentazione prodotta dal Tribunale Internazionale per i crimini di guerra in ex-Jugoslavia - e all'adozione di approcci diversi, tra i quali la storia locale e la microstoria.

Il volume consiste di otto capitoli, ognuno dei quali presenta il dibattito relativo a un aspetto specifico del conflitto e della riconciliazione/ricostruzione. Seguono una cronologia degli eventi che hanno anticipato, accompagnato e segnato la fine delle guerre jugoslave nel ventennio 1980-2000, e una bibliografia numerata e suddivisa per temi, volta a facilitare la consultazione e l'approfondimento degli aspetti trattati nel volume. Lo scopo principale dell'autrice è invitare il lettore a mettere in dubbio interpretazioni e narrazioni nelle quali etnicità e nazionalismo si propongono di spiegare in toto le guerre jugoslave. Baker mostra i limiti analitici e le pericolose generalizzazioni che possono derivare dall'uso sommario di queste categorie. Come correttivo di tali tendenze auspica l'analisi delle forme di protesta dal basso che presero piede negli anni Ottanta e Novanta e che, articolando il disagio sociale ed economico di quegli anni senza legarlo a rivendicazioni etniche o nazionali, sono rimaste trascurate. 


\section{REPE, Božo, Milan Kučan, prvi predsednik [Milan Kučan, il primo presidente], Modrijan, Ljubljana, 2015, 616 pp.}

a cura di Štefan ČOK

Milan Kučan, prvi predsednik rientra nel novero dei libri che in assoluto hanno fatto maggiormente discutere in Slovenia nel corso dell'ultimo anno. L'autore, Božo Repe, è professore ordinario di storia contemporanea presso l'Università di Lubiana, Milan Kučan è stato il primo presidente della Slovenia indipendente. L'opera è il frutto della collaborazione fra $\mathrm{i}$ due che ha preso avvio già negli anni Novanta, con la pubblicazione del libro di Božo Repe Jutrije nov dan e che li ha visti collaborare anche nell’impegno nel Forum 21, organizzazione della società civile fondata nel 2004. L'opera si concentra soprattutto sull'attività politica di Kučan, dedicando solo la parte introduttiva agli aspetti personali. La seconda, terza e quarta parte trattano il periodo in cui Kučan è stato ai vertici della politica slovena, dapprima negli ultimi anni della Jugoslavia poi come primo presidente della Slovenia indipendente, all'attività politica di Kučan dal termine del suo mandato presidenziale nel 2002 a oggi è infine dedicata l'ultima parte. Proprio sul periodo della transizione è posta la maggiore attenzione: l'autore presenta l'operato di Kučan sulla base di una ricca documentazione archivistica, dei volumi usciti negli ultimi vent'anni sull'argomento e delle numerose testimonianze dei protagonisti, parte delle quali è stata fornita espressamente per quest'opera. Repe ha voluto sottolineare durante alcune presentazioni del libro che si tratta di un'opera pensata per il pubblico sloveno, trattandosi di argomenti che sono ancora materia di scontro e di polemica politica e quindi affrontati con una ricca base documentaria. Il materiale d'archivio utilizzato poggia principalmente sull'archivio personale di Kučan e sugli archivi delle istituzioni slovene; l'opera è impreziosita infine da una ricca documentazione fotografica, da una cronologia e da un indice dei nomi.

\section{HAJDARPASIĆ, Edin, Whose Bosnia? Nationalism and Political} Imagination in the Balkans, 1840-1914, London, Cornell University Press, 2015, 271 pp.

a cura di Fabio GIOMI

Ci vuole una certa audacia, nel 2015, a pubblicare un libro sulla Bosnia e la questione nazionale. Gli ultimi venti anni, infatti, sono stati caratterizzati da una vera e propria proliferazione di questo tipo di studi, tanto a livello internazionale che (post-) 
jugoslavo. Nel tentativo di comprendere e spiegare la dissoluzione della Jugoslavia, un numero importante di storici, politologi e sociologi ha cercato di approfondire il processo di nazionalizzazione della società bosniaca e di ricostruire il ruolo della Bosnia nei progetti nazionali delle élite locali, in particolare croate e serbe. Nonostante questo Edin Hajdarpasić, storico di origine bosniaca della Loyola University (Chicago), riesce nellimpresa di scrivere un testo innovativo. Non solo perché egli mostra di essere immune da quel "nazionalismo metodologico" che troppo spesso ha caratterizzato questo tipo di ricerche. Ciò che rende questo libro convincente è piuttosto il modo in cui la questione nazionale è abbordata: invece di analizzare le narrazioni nazionali singolarmente, l'autore organizza la propria riflessione attorno a cinque topoi: il popolo, la sofferenza, l'attivismo, la gioventù, l’impero. Così facendo Hajdarpasić mette in luce la circolazione di idee, i prestiti, le tensioni all'interno e fra dei differenti discorsi nazionali che, fra il 1840 e il 1914, hanno strutturato il dibattito su "di chi è la Bosnia". L'autore introduce poi un nuovo concetto, quello di ( $b r$ )other, una specifica figura delle differenti narrazioni nazionali che abbraccia ciò che è comune, e quindi "fratello", ma allo stesso tempo il suo opposto, il radicalmente "altro". Questo concetto aiuta a comprendere le continue oscillazioni delle élite nazionaliste locali fra politiche di assimilazione e di marginalizzazione dell'altro.

\section{KOVIĆ, Miloš (a cura di), Srbi 1903-1914. Istorija ideja [I serbi 19o3-1914. Storia delle idee], Beograd, Clio, 2015,861 pp.}

a cura di Fabio GIOMI

Il volume curato da Miloš Ković, storico della facoltà di Filosofia di Belgrado, ha un obiettivo ambizioso: offrire uno spaccato delle principali culture politiche che circolavano nell'élite serba durante il regno di Petar I Karađorđević. Il periodo che va dal 1903, anno di insediamento del nuovo re, allo scoppio della Grande Guerra rappresenta un momento di estrema importanza per la storia di questo paese, caratterizzato da nuove alleanze internazionali, dalla fiducia in una imminente unificazione nazionale e in una progressiva liberalizzazione della vita politica. Mai come in questo periodo l'élite serba si è sentita in contatto con le idee che venivano da "fuori”, in particolare dall'Europa occidentale e dalla Russia. Il volume è strutturato in due sezioni, la prima dedicata alle culture politiche che circolavano nello spazio pubblico serbo, e la seconda consacrata alle personalità che hanno contribuito ad elaborarle, arricchirle e diffonderle. L'obiettivo, reso esplicito nell'introduzione, è quello di scrivere una storia delle idee della nazione serba. È interessante notare come 
- segno dei tempi! - i capitoli più lunghi siano dedicati a idee come "democrazia" (116 pagine) e "liberalismo" (49 pagine), che il "femminismo" (29 pagine) entri a pieno titolo fra le culture politiche strutturanti dell'epoca, e che lo "jugoslavismo" abbia ormai un posto tutto sommato ridotto (10 pagine). La seconda parte, quella legata alle personalità, ha certamente il limite di includere - ancora! - solo uomini fra le grandi figure nazionali, ignorando le donne che proprio in quel periodo erano sempre più visibili nello spazio pubblico. Un tratto positivo è quello di considerare anche personalità che vivevano al di fuori dei confini dello stato, come Nikola Tesla, mostrando come la storia delle idee serba diventi intelligibile solo se inclusa in spazi di circolazione più vasti.

\section{BUTUROVIĆ, Amila, Carved in Stone, Etched in Memory. Death,} Tombstones and Commemoration in Bosnian Islam since c.15oo, Farham, Ashgate Publishing, 2015, 256 pp.

a cura di Monica PRIANTE

La monografia di Amila Buturović approfondisce le tradizioni commemorative e i monumenti funebri Islamici in Bosnia dal 1500. Il processo di transizione tra il preOttomano, sotto l'autorità Cristiana, al sistema culturale nell'impero Ottomanoislamico portò ad una confluenza di differenti stili funerari che riflettevano la situazione creatasi tra un numero sempre crescente di conversioni e una nuova sensibilità escatologica. In questo periodo di trasformazioni, avvenute dopo l'incorporazione della Bosnia nell'Impero Ottomano, anche la morte iniziò ad essere "islamizzata" e commemorata con l'aiuto di un nuovo dizionario epigrafico ed iconografico. In particolare, utilizzando le iscrizioni epigrafiche, l'autrice analizza le modalità con cui la collettività abbandonò progressivamente le pratiche precedenti e il modo in cui si continuarono a ricordare i propri avi in un processo di adattamento e convergenza di differenti sistemi escatologici. Dalla ricerca emerge che l'islamizzazione delle pratiche commemorative seguì con un ritmo molto più lento il processo di ottomanizzazione in atto in altri segmenti della società. L'autrice, infatti, sostiene che soltanto tra il XVIII e il XIX secolo le pratiche commemorative arrivarono a riflettere una netta separazione tra i modelli Cristiani e quelli Islamici, consolidando (e confermando) il sentimento di differenziazione tra i diversi gruppi identitari. Oltre a questo, definendo le lapidi "guardiani della memoria", l'autrice insiste anche sul ruolo di mediazione delle lapidi nel rapporto tra morti, vivi e la memoria che li collega. Infine, lo studio si concentra sulla visibilità e l'impatto che le pratiche funerarie 
avevano (e hanno) su di una comunità, per aiutarci a riflettere sul legame di continuità tra passato, presente e futuro

DUDA, Igor, Danas kada postajem pionir: Djetinjstvo i ideologija jugoslavenskoga socijalizma [Oggi che sono diventato pioniere. Infanzia e ideologia del socialismo jugoslavo], Zagreb, Srednja Europa, 2015, 274 pp.

a cura di Francesca ROLANDI

Danas kada postajem pionir: Djetinjstvo i ideologija jugoslavenskoga socijalizma di Igor Duda, docente presso l'Università di Pola, affronta il tema dell'associazionismo giovanile in Jugoslavia come parte del progetto pluriennale "La creazione dell'uomo socialista. La società croata e l'ideologia del socialismo jugoslavo", sviluppato nell'ambito del Centro per gli studi culturali e storici sul socialismo. Duda, iniziatore degli studi sul tempo libero in Croazia, si focalizza in questo volume sulla Lega dei pionieri, organizzazione di massa e primo livello del progetto di creazione di un individuo su misura del socialismo jugoslavo, attraverso l'organizzazione del suo tempo libero. La Lega dei pionieri, nata durante la guerra di liberazione, si propone di offrire ai giovani un modello educativo - concorrenziale rispetto a quello familiare ma soprattutto rispetto agli agenti educativi tradizionali come la chiesa cattolica - che stimolasse il patriottismo, il rispetto per i miti costitutivi della Jugoslavia socialista, ma anche valori considerati cruciali per sviluppare una libera personalità. Tra questi numerosi aspetti e contraddizioni, il volume di Duda esplora come la Lega dei pionieri si sia confrontata con questioni cruciali quali lo scontro tra Tito e Stalin, l'ideologia jugoslava come soggetto in divenire, l'autogestione, la fratellanza e unità, lo scontro tra modernità e tradizione, la nascente società dei consumi, la cultura di massa occidentale, la comunità italiana in Istria, fino alla dissoluzione della Federazione jugoslava. Quello che emerge è un tentativo di incasellare il tempo libero delle giovani generazioni che tuttavia convive con una politica educativa emancipatoria e un'attenzione all'infanzia come incubatrice del futuro.

DONIA, Robert J., Radovan Karadžić. Architect of the Bosnian Genocide, Cambridge, Cambridge University Press, 2015, 339 pp.

a cura di Alfredo SASSO 
Robert Donia, già autore di testi fondamentali sulla Bosnia-Erzegovina contemporanea, dedica alla figura di Radovan Karadžić uno studio solido e innovativo, che indaga "non solo la sua vita, ma anche le dinamiche interne che guidarono i nazionalisti serbo-bosniaci a commettere atrocità di massa, compreso il genocidio”. Ne emerge un profilo fluido, che si discosta da determinismi e caricature. Prima del 1990 Karadžić "non mostrò un profilo pubblico nazionalista": fu per un incrocio di opportunità, contatti personali, e un vago risentimento anticomunista, che entrò in politica e scalò i vertici del nazionalismo serbo-bosniaco. Iniziò così la sua metamorfosi da "nazionalista naif" e conciliante verso gli avversari politici a "pianificatore visionario" del conflitto etnico a partire dall'autunno 1991. Cruciale è la ricostruzione del rapporto con la Serbia di Slobodan Milošević, tutt'altro che subalterno: l'iniziale "combinazione di collegialità e competizione" lasciò spazio a insanabili divergenze tattiche e strategiche. Karadžić era ossessionato dalla statualità serbo-bosniaca da perseguire a ogni costo, compresi gli atti di pulizia etnica e genocidio, la cui pianificazione politica è ben documentata nel testo. Milošević era calcolatore, manipolatore, preoccupato per il suo potere e per le reazioni internazionali; "spesso descritto come un tirannico assassino di massa, ma qui emerge come un leader indeciso e debole". Le fonti sono un altro punto di forza, trattandosi di una ingente mole di trascrizioni telefoniche, documenti istituzionali e di partito in possesso del Tribunale Internazionale per l'ex-Jugoslavia (ICTY) di cui Donia è stato consulente, che risultano ancora poco utilizzati dagli studiosi benché siano in buona parte accessibili liberamente tramite l'archivio online ICTY. 


\section{* Gli autori}

Marco Abram ha conseguito il Dottorato di ricerca presso l'Università di Udine ed è stato visiting $\mathrm{PhD}$ student presso l'Università di Belgrado. Si occupa soprattutto di storia culturale della Jugoslavia socialista. Tra il 2013 e il 2014 ha lavorato come ricercatore presso Osservatorio Balcani e Caucaso, mentre nel 2015 è stato research fellow al Center for Advanced Studies South Eastern Europe. Attualmente è research fellow presso il Dipartimento di Storia dell'Università di Fiume.Ha fatto parte della redazione di «Diacronie. Studi di Storia contemporanea» dal 2009 al 2013.

URL: < http://www.studistorici.com/progett/autori/\#Abram >

Sara Bernard ha conseguito il Dottorato di ricerca in Storia del Sud-est Europa presso l'Università di Ratisbona con una ricerca sulle migrazioni di ritorno nella Jugoslavia socialista. I suoi interessi di ricerca riguardano principalmente dinamiche politiche e socio-economiche legate alle migrazioni internazionali che hanno caratterizzato le interazioni tra paesi socialisti e/o in via di sviluppo e l'Europa occidentale durante la guerra fredda.

URL: < http://www.studistorici.com/progett/autori/\#Bernard >

Štefan Čok consegue la laurea in Storia contemporanea e la laurea specialistica in Storia della Società e della Cultura contemporanea presso l'Università degli Studi di Trieste. Nel 2015 conclude il proprio dottorato di ricerca in Storia dell'Europa e del Mediterraneo presso l'Università del Litorale di Koper-Capodistria, Slovenia, con un lavoro sulle elites liberalnazionali italiane triestine a cavallo fra XIX e XX secolo. Collabora con lo SLORI-Istituto Sloveno di Ricerche e la Sezione Storia ed Etnografia della Biblioteca nazionale slovena e degli studi di Trieste. Negli ultimi anni ha partecipato a vari progetti legati alla trasmissione della storia e delle memorie alle giovani generazioni, con particolare attenzione all'area altoadriatica e ai rapporti fra le diverse comunità che la popolano.

URL: < http://www.studistorici.com/progett/autori/\#Cok >

Fabio Giomi (CNRS) è ricercatore presso il Centre d'Études Turques, Ottomanes, Balkaniques et Centrasiatiques (CNRS, EHESS, Collège de France) a Parigi. Si occupa di storia sociale, culturale e politica dello spazio jugoslavo dalla fine del XIX alla metà del XX secolo.

URL: < http://www.studistorici.com/progett/autori/\#Giomi > 
Monica Priante, dottore di ricerca in storia moderna e contemporanea presso i Hrvatski studiji (Università di Zagabria), tema della ricerca i rapporti tra la Chiesa ortodossa e cattolica nel XIX secolo e l'operato del vescovo croato Josip Juraj Strossmayer in Serbia. Successivamente, le sue ricerche si sono concentrate sulla storia culturale italiana ed europea nell'Ottocento, specialmente sulla ricezione della simbologia del Risorgimento italiano in Croazia centrale e Serbia. Negli ultimi anni, i suoi interessi di studio si sono spostati alla tanatologia culturale, con particolare attenzione al legame tra la storia della morte e la costruzione delle identità nazionali in Croazia tra il XIX secolo e il XX secolo. Attualmente collabora come docente a contratto con l’Università Cattolica di Zagabria.

URL: < http://www.studistorici.com/progett/autori/\#Priante >

Francesca Rolandi ha conseguito il dottorato di ricerca in Slavistica presso l'Università degli studi di Torino nel 2012 con una tesi incentrata sull'influenza della cultura di massa italiana in Jugoslavia, vincitrice nel 2014 del premio Vinka Kitarović. Attualmente è research fellow presso l'Università di Fiume nell'ambito del programma Newfelpro con un progetto sui richiedenti asilo jugoslavi in Italia durante la guerra fredda.

URL: < http://www.studistorici.com/progett/autori/\#Rolandi >

Alfredo Sasso è borsista post-doc presso il CAS-Università di Rijeka (2016), dottore di ricerca in Storia Contemporanea presso l'Università Autonoma di Barcellona (2015). I suoi interessi di ricerca si concentrano sui movimenti politici nello spazio jugoslavo e post-jugoslavo contemporaneo. Ha svolto attività di ricerca in Bosnia-Erzegovina, Serbia, Croazia e Messico. È redattore della rivista accademica spagnola «Tiempo Devorado» e del magazine italiano «East Journal».

URL: < http://www.studistorici.com/progett/autori/\#Sasso > 


\section{Per citare questo articolo:}

ABRAM, Marco, BERNARD, Sara, ČOK, Štefan, GIOMI, Fabio, PRIANTE, Monica, ROLANDI, Francesca, SASSO, Alfredo, «Panoramica: Area post-jugoslava 2015», Diacronie. Studi di Storia Contemporanea : La voce del silenzio: intelligence, spionaggio e conflitto nel XX secolo, 29/12/2016,

URL:< http://www.studistorici.com/2016/12/29/jugoslavia_numero_28/ >

Diacronie Studi di Storia Contemporanea $\beta$ www.diacronie.it

Risorsa digitale indipendente a carattere storiografico. Uscita trimestrale. redazione.diacronie@hotmail.it

Comitato di redazione: Jacopo Bassi - Luca Bufarale - Antonio César Moreno Cantano - Deborah Paci - Fausto Pietrancosta - Alessandro Salvador - Matteo Tomasoni - Luca Zuccolo

Diritti: gli articoli di Diacronie. Studi di Storia Contemporanea sono pubblicati sotto licenza Creative Commons 3.0. Possono essere riprodotti e modificati a patto di indicare eventuali modifiche dei contenuti, di riconoscere la paternità dell'opera e di condividerla allo stesso modo. La citazione di estratti è comunque sempre autorizzata, nei limiti previsti dalla legge. 